\title{
Challenges of treating autoimmune diseases in patients presenting with immunodeficient conditions
}

\begin{abstract}
Common variable immunodeficiency (CVID) is a rare disorder; however, it is the most documented primary immunodeficiency in the USA. Although recurrent sinopulmonary infections occur in up to $80 \%$ of patients, autoimmune disorders are surprisingly common with this type of immunodeficiency. Autoimmune diseases, the most common being immune thrombocytopenic purpura and hemolytic anemia, affect approximately $25 \%$ of all patients with CVID. Furthermore, aseptic arthritis, a disease that resembles rheumatoid arthritis (RA), is particularly common and occurs in $1 \%-10 \%$ of patients with CVID; however, RA itself occurs less frequently. Herein, we report a case of CVID and RA with challenges in using immunosuppressive therapy in conditions of immunodeficiency and presence of severe recurrent infections.
\end{abstract}

Keywords: common variable immunodeficiency, rheumatoid arthritis, autoimmune diseases, immunosuppressive therapy, recurrent infections
Volume 9 Issue 2 - 202I

\author{
Ahmed M Ahmed,' Dima Youssef,' Mohamed \\ Mohamed $^{2}$ \\ 'Department of Infectious, Inflammatory and Immunologic \\ Diseases, East Tennessee State University, USA \\ ${ }^{2}$ Rheumatology division, Kings daughter medical center, USA
}

Correspondence: Dima Youssef, East Tennessee State

University, Quillen College of Medicine, Veteran Affairs Building

3, Box 70622, Johnson City, Tennessee 376I4, USA,

Tel (423) 439-805I, Email youssef@etsu.edu

Received: May 2I, 202I | Published: May 3I, 202 I

\section{Case presentation}

A 29-year-old man was diagnosed with common variable immunodeficiency (CVID) in 2012. The patient was administered monthly intravenous immunoglobulin therapy that was complicated by bronchiectasis due to recurrent pulmonary infections. One year later, the patient complained of nonspecific fatigue and symmetric multiple upper and lower limb joint pain and swelling. The pain involved the shoulders, elbows, wrists, metacarpophalangeal joints (MCPs), and knees for 3 months. In addition, the patient described daily morning stiffness. The patient's vital signs were within normal ranges. Physical examination revealed swelling, redness, warmth, and tenderness involving bilateral wrists and multiple MCPs. X-ray images showed periarticular osteopenia of the MCPs compatible with early inflammatory arthritis. The serological panel showed a negative rheumatoid factor and anti-cyclic citrullinated peptide. Ultrasound examination of the elbows showed active synovitis, which confirmed the diagnosis of seronegative RA. At that time, the patient was commenced on sulfasalazine, methotrexate, and hydroxychloroquine for 1 year without improvement. Given worsening arthritis and evidence of erosions on repeat hand X-ray, the patient was deemed to have failed triple therapy, and the decision to escalate the patient's immunosuppressive therapy was made. After a discussion with the immunology team, the patient was started on etanercept without improvement in his symptoms. He was then switched to adalimumab with minimal improvements in his symptoms; however, unfortunately, the patient developed recurrence of severe infections necessitating frequent hospitalizations and interruption of adalimumab treatment. Meanwhile, the patient developed recurrent severe flares of RA, which were treated with steroids.

\section{Discussion}

CVID is a primary immunodeficiency disorder, the main feature of which is B cell differentiation with defects in immunoglobulin production. It is a combination of hypogammaglobulinemia secondary to multiple genetic defects. It is the most common type of severe antibody deficiency in both children and adults. ${ }^{1}$ The word "variable" describes the clinical manifestations and heterogenicity of the disease, which include recurrent infections, chronic autoimmune disorders, gastrointestinal disease, lung disease, and increased susceptibility to lymphoma.

Autoimmune disorders can present with other diseases, with hematologic disorders being the most prevalent autoimmune disorders in patients with CVID. ${ }^{2,3}$ Immune dysregulation is considered as the cause of autoimmunity in patients with CVID, but the specific mechanisms associated with it remain unknown. Patients with CVID and autoimmune disorders do not show a trend of developing recurrent infections due to unclear reasons; however, they are susceptible to enteropathy development. ${ }^{4,5} \mathrm{RA}$ is an autoimmune systemic disorder of unknown etiology that presents typically as symmetric inflammatory polyarthritis. It characteristically affects MCPs and proximal interphalangeal joints of the hands and feet. It can lead to joint destruction, deformity, and eventually loss of function if inadequately treated or if the patient is resistant to treatment. The patient in this case report was diagnosed with CVID in 2012 at an outside facility. His disease was complicated by bronchiectasis due to recurrent pulmonary infections despite adequate monthly intravenous immunoglobulin (IVIG) therapy. One year later, RA was diagnosed based on the clinical presentation and imaging that showed RA changes.

Studies have suggested treating patients with CVID and aseptic arthritis with IVIG therapy and administering a treatment identical to that received by individuals without immunodeficiency to treat concurrent RA; this is performed if treatment with immunoglobulins fails. However, practically, this may be challenging in patients who continue experiencing recurrent infections requiring frequent hospitalizations, despite receiving adequate doses of IVIG therapy. Therefore, such patients cannot tolerate immunosuppressive therapy designed to achieve remission of erosive RA, which is similar to the patient presented in this case report. In such cases, the only practical solution is a high dose of steroids for acute flares and a maintenance therapy with a low dose of steroids to prevent recurrence. 


\section{Conclusion}

Utilization of biologic agents to treat autoimmune disorders has become increasingly prevalent which considerably suppresses immune response and increments risks of both immunodeficiency flares as well as infections. It is imperative to consider the balance between the risks and benefits of aggressive immunosuppressive therapy when treating complex autoimmune diseases such as CVID and RA.

\section{Acknowledgments}

None.

\section{Conflicts of interest}

The authors declare that there is no conflict of interest.

\section{References}

1. Primary immunodeficiency diseases in America. The third national survey of patients. 2007.

2. Boileau J, Mouillot G, Gérard L, et al. Autoimmunity in common variable immunodeficiency: correlation with lymphocyte phenotype in the French DEFI study. J Autoimmun. 2011;36:25-32.

3. Wang J, Cunningham-Rundles C. Treatment and outcome of autoimmune hematologic disease in common variable immunodeficiency (CVID). $J$ Autoimmun. 2005;25:57-62.

4. Resnick ES, Moshier EL, Godbold JH, et al. Morbidity and mortality in common variable immune deficiency over 4 decades. Blood. 2012;119:1650-1657.

5. Gathmann B, Mahlaoui N, Ceredih, et al. Clinical picture and treatment of 2212 patients with common variable immunodeficiency. J Allergy Clin Immunol. 2014;134:116-126. 